\title{
Comunicação e relacionamento interpessoal no centro cirúrgico: aplicação da metodologia da problematização
}

\author{
Communication and interpersonal relationship in the surgical center: application of the \\ problematization methodology
}

Comunicación y relación interpersonal en el centro quirúrgico: aplicación de la metodología de la problematización

\begin{abstract}
Kezia Cristina Batista dos Santos ${ }^{1 *}$, Alinne Suelma dos Santos Diniz ${ }^{1}$, Josilma Silva Nogueira ${ }^{1}$, Isaura Letícia Tavares Palmeira Rolim ${ }^{1}$ Ana Hélia de Lima Sardinha ${ }^{1}$.
\end{abstract}

\section{RESUMO}

Objetivo: Relatar a experiência da aplicação da metodologia da problematização nas dificuldades de comunicação e relacionamento interpessoal entre os profissionais do centro cirúrgico de um hospital universitário. Detalhamento do caso: Aplicou-se o método do Arco de Maguerez com 68 profissionais da equipe cirúrgica: médicos, enfermeiros e técnicos de enfermagem de um centro cirúrgico adulto de um hospital universitário do nordeste do Brasil, no ano de 2017. Identificou-se como principais problemas: dificuldades no relacionamento entre os membros da equipe; desvalorização do trabalho do enfermeiro; comunicação deficiente ou falha na comunicação; formação de "guetos" profissionais e sobrecarga de trabalho e funções. Considerações Finais: O Arco de Maguerez mostrou-se uma metodologia ativa eficiente ao aproximar teoria e prática com a problematização da realidade observada. Identificou-se o desenvolvimento do pensamento crítico-reflexivo por parte da equipe cirúrgica e maior conscientização acerca da importância da manutenção de uma boa comunicação e relacionamento interpessoal entre os profissionais que ali atuam, o que contribuiu para fortalecimento das relações entre equipe multiprofissional.

Palavras-chave: Comunicação, Educação em Enfermagem, Enfermagem de Centro Cirúrgico, Equipe de Assistência ao Paciente.

\begin{abstract}
Objective: To report the experience of applying the methodology of problematization in the difficulties of communication and interpersonal relationship among the professionals of the surgical center of a university hospital. Case detail: The Arch of Maguerez method was applied with 68 professionals from the surgical team: physicians, nurses and nursing technicians from an adult surgical center of a university hospital in the northeast of Brazil, in 2017. The main problems identified were: difficulties in the relationship between team members; devaluation of nurses' work; poor communication or communication failure; formation of professional "ghettos" and over load of work and functions. Final considerations: The Arch of Maguerez proved to be an efficient active methodology when approaching theory and practice with the problematization of observed reality. It was identified the development of critical-reflexive thinking by the surgical team and greater awareness about the importance of maintaining good communication and interpersonal relationship among the professionals who work there, which contributed to the strengthening of relationships among multiprofessional team.
\end{abstract}

Key words: Communication, Nursing Education, Operating Room Nursing, Patient Care Team.

${ }^{1}$ Universidade Federal do Maranhão (UFMA). São Luís-MA E-mail: kezia cristinabs@hotmail.com SUBMETIDO EM: 4/2019 ACEITO EM: 5/2019 PUBLICADO EM: 6/2019 


\section{RESUMEN}

Objetivo: Informar la experiencia de la aplicación de la metodología de la problematización en las dificultades de comunicación y relación interpersonal entre los profesionales del centro quirúrgico de un hospital universitario. Detalle del caso: Se aplicó el método del Arco de Maguerez con 68 profesionales del equipo quirúrgico: médicos, enfermeros y técnicos de enfermería de un centro quirúrgico adulto de un hospital universitario del nordeste de Brasil, en el año 2017. Se identificó como principales problemas: dificultades en la relación entre los miembros del equipo; desvalorización del trabajo del enfermero; comunicación deficiente o falla en la comunicación; formación de "guetos" profesionales y sobrecarga de trabajo y funciones. Consideraciones finales: El Arco de Maguerez se mostró una metodología activa eficaz al aproximar teoría y práctica con la problematización de la realidad observada. Se identificó el desarrollo del pensamiento críticoreflexivo por parte del equipo quirúrgico y mayor concientización acerca de la importancia del mantenimiento de una buena comunicación y relación interpersonal entre los profesionales que allí actúan, lo que contribuyó para el fortalecimiento de las relaciones entre el equipo multiprofesional.

Palabras clave: Comunicación, Educación em Enfermería, Enfermería de Quirófano, Grupo de Atención al Paciente.

\section{INTRODUÇÃO}

O centro cirúrgico (CC) é uma unidade que apresenta cuidados de alta complexidade e com uso de tecnologia avançada. Os profissionais de enfermagem que atuam no centro cirúrgico são responsáveis pelo cliente da admissão à alta do setor, respeitando sua individualidade, prestando uma assistência adequada à necessidade do paciente (ALVES NB e ARAUJO GF, 2015).

Devido à complexidade deste ambiente e frequente estresse vivenciado pela equipe cirúrgica no dia-a-dia de serviço, conflitos podem emergir, tornando o ambiente de trabalho tenso e desgastante induzindo dificuldades relacionais entre a equipe. Para gerir tais conflitos é importante a utilização de ferramentas administrativas, da comunicação, da negociação e do trabalho em equipe. A literatura aponta diversas formas de gerenciar/solucionar conflitos, dentre elas: acomodação; dominação; barganha/compromisso; solução integrativa de problemas (CIAMPONE MHT e KURCGANT P, 2005); diálogo (CORRADI EM, et al., 2008), escuta (AMESTOY SC, et al., 2014), negociação (MARTA CB, et al., 2010; CORRADI EM, et al., 2008). Nesse percurso, torna-se importante que os profissionais inter-relacionem-se de tal maneira que possam alcançar objetivos comuns e dinâmica de trabalho harmoniosa (OLIVEIRA MAN e SANTA ROSA DO, 2016).

A comunicação e o trabalho em equipe são determinantes na qualidade da assistência ao paciente. $A$ comunicação eficaz é essencial para o bom desenvolvimento do trabalho, pois configura-se elo de interação que fortalece o vínculo entre a equipe interdisciplinar e o cliente (NOGUEIRA JWS e RODRIGUES MCS, 2015).

No CC as relações profissionais são mais intensas e os profissionais tornam-se mais próximos uns dos outros, o que acaba tornando o ambiente propício a relações conflituosas. As relações interpessoais no CC são caracterizadas como hierárquicas e verticalizadas entre as diferentes categorias profissionais, podendo resultar em sentimento de desvalorização no reconhecimento profissional, que associados à remuneração inadequada e jornada de trabalho exaustiva acabam por refletir nas relações entre a equipe e, consequentemente, na qualidade da assistência prestada ao usuário, bem como no bem-estar psíquico dos profissionais (TRAJANO MCF, et al., 2017).

No cotidiano de trabalho no CC no campo de estudo analisado, observou-se, pelos profissionais residentes, a existência de conflitos, falha na comunicação entre a equipe cirúrgica e dificuldades no relacionamento interpessoal. Assim, ao considerar a presença de alguns conflitos, questionou-se: quais os desafios enfrentados pela equipe do CC na comunicação e nos relacionamentos interpessoais? Diante disto, este estudo objetivou relatar a experiência do uso da metodologia da problematização nas dificuldades de comunicação e relacionamento interpessoal entre os profissionais do centro cirúrgico. Aplicou-se o método 
do Arco de Maguerez com 68 profissionais da equipe cirúrgica: médicos, enfermeiros e técnicos de enfermagem de um centro cirúrgico adulto de um hospital universitário do nordeste do Brasil, no ano de 2017.

O método do arco de Charles Maguerez é o principal referencial teórico para a metodologia da problematização e é composto por cinco etapas: 1 . Observação da realidade, realizada a partir da observação sistemática e registro da realidade vivenciada e identificação do(s) problema(s); 2 . Pontos-chave, a partir da definição do(s) problema(s) definem-se os tópicos a serem estudados, estabelecendo os prioritários e essenciais; 3. Teorização, consiste na busca de informações acerca do(s) problema(s), a partir de fundamentação teórica, servindo de base para explicá-lo, assim como seu impacto na realidade observada; 4. Hipótese de solução, etapa de análise e reflexão com formulação de hipóteses que apontem o caminho para a resolução do problema; 5. Aplicação à Realidade, nesta etapa, são aplicadas as propostas de soluções para o(s) problema(s) identificado(s), buscando a transformação da realidade (BERBEL NAN, 1998).

Por se tratar de um estudo observacional, do tipo relato de experiência, não houve necessidade da submissão deste estudo a um Comitê de Ética em Pesquisa (CEP), pois o mesmo objetiva descrever a experiência das autoras no campo de atuação supracitado originado de vivências e experiências oriundas da prática profissional e sem identificação dos participantes do estudo garantindo-lhes privacidade, confidencialidade dos dados e respeito à dignidade humana, estando de acordo com a resolução 466/2012 do Conselho Nacional de Saúde.

\section{DETALHAMENTO DO CASO}

A população do estudo correspondeu a 138 profissionais do centro cirúrgico adulto de um hospital universitário do nordeste brasileiro. As atividades foram desenvolvidas junto à 68 profissionais, convidados conforme disponibilidade, escala de serviço e demanda cirúrgica que aceitaram participar dos encontros realizados com a equipe, a saber: 4 enfermeiros e 16 médicos residentes, 9 enfermeiros, 27 técnicos de enfermagem e 12 médicos. Os encontros aconteceram nas terças e quintas-feiras, com duração de quatro horas, no período da tarde, durante o mês de dezembro de 2017.

Os dados foram coletados por meio de observação direta do cotidiano de trabalho da equipe cirúrgica e a partir de discussões, rodas de conversa e seminários realizados com os profissionais durante os encontros. A análise dos dados foi realizada por similaridade temática a partir do relato dos profissionais e por meio dos principais pontos-chave identificados na etapa 2 do método do arco de Maguerez.

A descrição da experiência é apresentada nas cinco etapas do arco de Maguerez descritas a seguir:

\section{Primeira etapa: Observação da Realidade}

Nesta primeira etapa do arco de Maguerez observou-se a realidade do setor e realizou-se o levantamento dos principais problemas relacionados às dificuldades na comunicação e ao relacionamento interpessoal entre os profissionais no centro cirúrgico.

Inicialmente as pesquisadoras realizaram a coleta de dados de forma observacional, com o levantamento de informações sobre a rotina do setor e dinâmica de trabalho da equipe cirúrgica, durante 15 dias úteis anteriores ao início dos encontros com os profissionais, compreendendo o período de 10 a 30 de novembro de 2017, durante o horário de treinamento em serviço (TS) dos profissionais residentes (07h da manhã a 19h da noite).

Nesta etapa, identificou-se a ocorrência frequente de situações conflituosas e dificuldades na relação entre equipe multiprofissional durante a rotina do serviço, além de falha/dificuldade na comunicação entre os profissionais dentro das salas de cirúrgica e durante a passagem de turno.

Posteriormente, conforme as etapas seguintes do Arco de Maguerez e a partir do que foi percebido sobre a realidade em questão, os profissionais foram convidados a participar dos encontros em equipe multidisciplinar. A interação entre os pesquisadores e profissionais do setor se deu de forma colaborativa, por meio de contínua troca de conhecimentos e problematização da realidade vivenciada. 


\section{Segunda etapa: Pontos-Chave}

$\mathrm{Na}$ segunda etapa as pesquisadoras identificaram os pontos-chave a serem estudados e discutidos, no qual se embasaria a resolução da situação problema. Os pontos-chave foram levantados após a síntese das informações colhidas na etapa anterior, onde foram definidos os principais aspectos que precisavam ser compreendidos para buscar alternativas de resposta (hipóteses de solução) ao problema.

Assim, foram identificados como pontos-chave os principais problemas: 1. Dificuldades enfrentadas no relacionamento com a equipe (cirurgiões, anestesistas, técnicos de enfermagem); 2. Desvalorização do trabalho do enfermeiro; 3. Comunicação deficiente ou falha na comunicação; 4. Formação de "guetos" profissionais; 5 . Sobrecarga de trabalho e funções.

Diante dos pontos-chave encontrados e após discussão com a equipe cirúrgica identificou-se como possíveis causas, diferenças individuais de cada profissional; dimensionamento inadequado de trabalhadores à demanda de atividades e funções; rotina do setor (pressão nosocomial); corporativismo, cultura organizacional vertical, sobrecarga de trabalho e tendência a formação de guetos profissionais.

\section{Terceira etapa: Teorização}

Nessa terceira etapa, os profissionais presentes na unidade conforme escala de serviço, foram reunidos em roda de conversa para estudarem e levantarem discussões sobre o tema: comunicação e relacionamento interpessoal.

Nesta etapa, os encontros, ocorreram com a equipe cirúrgica em três momentos, onde foram realizadas dinâmicas de aproximação e discussão de conteúdos sobre a importância da comunicação e manutenção de um bom relacionamento entre equipe multiprofissional. As pesquisadoras decidiram dividir esta etapa em três momentos, com a finalidade de reunir o maior número possível de profissionais da equipe nos encontros. Durante a reunião, os profissionais realizaram leituras a partir de folders informativos e artigos científicos que evidenciassem o tema; assistiram vídeos sobre a temática, entrevistas e mini seminários sobre o assunto, consultas de especialistas da área e discutiram o assunto assimilado em grupo.

Durante os encontros com a equipe foi evidenciado que os problemas de comunicação e relacionamento interpessoal estavam ligados, principalmente, a singularidade dos sujeitos, grande número e alta rotatividade de profissionais e pela própria complexidade do ambiente cirúrgico, que por vezes pode comprometer a comunicação entre os profissionais.

\section{Quarta etapa: Hipóteses de Solução}

Neste momento, com os conhecimentos aprimorados através das discussões e levantamentos realizados sobre a importância da comunicação e do relacionamento interpessoal, foram elaboradas, de maneira crítica e criativa, as possíveis soluções para a manutenção de um processo de comunicação eficiente e melhora do relacionamento interpessoal. Em contraposição à fase anterior, as hipóteses foram desenvolvidas rapidamente, já que estão intimamente ligadas a identificação dos pontos-chave.

As hipóteses formuladas para os questionamentos iniciais remeteram-se a: 1. Melhora na comunicação e no relacionamento interpessoal entre os profissionais; 2. Estimular a resolução de conflitos com diálogo participativo e; 3. Aprimoramento do convívio no setor.

\section{Quinta etapa: Aplicação da Realidade}

Para a resolução do problema, a equipe se propôs a realizar breves encontros antes do início do plantão como forma de aproximar e estreitar vínculos. Durante tais encontros, os profissionais conversavam, realizavam exercícios de alongamentos com objetivo de relaxamento e preparação para a rotina de trabalho. Foram realizadas dinâmicas quebra-gelo como forma de descontração e interação entre os profissionais, que se mostraram participativos. Discutiu-se com a líder do setor a necessidade de implantação de ações de educação permanente com a equipe cirúrgica no intuito de se manter e perpetuar as medidas implementadas com vistas a uma melhor comunicação e consequentemente melhor relacionamento interpessoal multiprofissional. 
Após a implementação das atividades e ações sugeridas pelos pesquisadores e pactuadas com toda a equipe, pode-se verificar que a aplicação da metodologia da problematização com o Arco de Maguerez contribuiu para compreensão e percepção crítica-reflexiva do problema vivenciado pelos profissionais, promovendo melhora na comunicação e na forma de interação e relacionamento entre os mesmos, com mudança da realidade observada.

\section{DISCUSSÃO}

Para discussão das dificuldades vinculadas a comunicação e ao relacionamento interpessoal entre os profissionais no centro cirúrgico, os resultados identificados na etapa 2 "pontos-chave" foram organizados em três aspectos por similaridade temática, a saber: dificuldades enfrentadas no relacionamento entre equipe multiprofissional; relação conflituosa entre equipe de enfermagem e corpo médico e; comunicação deficiente ou falha na comunicação entre os profissionais.

Atualmente, um dos desafios para garantia da segurança do paciente dentro do contexto hospitalar consiste em dar ênfase a comunicação efetiva como meta a ser alcançada pela equipe, assim como, proporcionar um ambiente de trabalho harmonioso com minimização de riscos e danos ao paciente (NOGUEIRA JWS e RODRIGUES MCS, 2015).

Como identificado na etapa 2, a partir da observação das pesquisadoras e após discussão com a equipe cirúrgica, foram apontadas como possíveis causas para os problemas na comunicação e relacionamento interpessoal no CC: diferenças individuais de cada profissional; dimensionamento inadequado de trabalhadores à demanda de atividades e funções; rotina do setor (pressão nosocomial); corporativismo, cultura organizacional vertical, sobrecarga de trabalho e tendência a formação de guetos profissionais. Os problemas decorrentes de tais aspectos já amplamente relatados na literatura, propiciam a ocorrência de conflitos (ALVES NB e ARAUJO GF, 2015; OLIVEIRA MAN e SANTA ROSA DO, 2016; TRAJANO MCF, et al., 2017; MARTINS CCF, et al., 2014; AMESTOY SC, et al., 2014; SANTOS PS, et al., 2015).

Diante da complexidade do trabalho em saúde e particularidades do CC, conflitos podem surgir entre as diversas categorias profissionais (AMESTOY SC, et al., 2014). O enfermeiro como líder da equipe de enfermagem e membro da equipe multidisciplinar presente no CC estar suscetível a conflitos, pois estabelece relacionamentos de caráter complexo com todos os profissionais da equipe, e que exigem habilidades eficazes para o controle de situações que muitas vezes ultrapassam o vínculo pessoal (MARTINS CCF, et al., 2014; CARVALHO R e BIANCHI ERF, 2016).

A existência de um conflito implica a desorganização de todos os integrantes da equipe multidisciplinar, além disso, repercute no aumento do estresse, devido à falta de participação na tomada de decisão, falta de apoio da gerência, sobrecarga de trabalho e rápidas mudanças de cunho tecnológico (SANTOS PS, et al., 2015).

Quanto à relação entre médicos e a equipe de enfermagem, pesquisadoras observaram que os cirurgiões geralmente apresentaram-se estressados, interferindo na rotina das salas operatórias, causando conflitos com o técnico de enfermagem circulante de sala e o enfermeiro, tais agravantes, consequentemente, se acumulavam nos enfermeiros, deixando-os cansados, desmotivados, levando-os ao estresse.

É comum se observarem conflitos entre as categorias profissionais, sendo mais frequente entre médicos e a equipe de enfermagem. Tais conflitos costumam interferir no desenvolvimento do trabalho em saúde podendo trazer repercussões negativas na qualidade da assistência aos usuários (SANTOS KCB, et al., 2018).

Dessa forma, o enfermeiro deve estar atento às características individuais dos diferentes profissionais, a fim de observar a origem dos conflitos. Utilizando como ferramenta, a escuta, o respeito e o diálogo, para administrar situações conflitantes e reduzir os impactos na assistência (AMESTOY SC, et al., 2014).

Em uma pesquisa que se avaliou os fatores que determinam a diferença de relação de poder entre os profissionais médicos e enfermeiros, identificou-se que conflitos entre a equipe multidisciplinar, principalmente 
entre estes profissionais, tendem a trazer reflexos na assistência ao paciente. Destacando a importância de uma boa relação, entre médicos e enfermeiros, como fator de prevenção de conflitos na equipe (SANTOS PS, et al., 2015).

O conflito entre médicos e enfermeiros, se instala devido a forma como é organizado o trabalho dos profissionais, em que se observa uma maior valorização e o domínio da classe médica. Tal fato, acarreta, muitas vezes, na desvalorização do trabalho do enfermeiro, em virtude da tentativa de solucionar problemas que não são de sua competência (AMESTOY SC, et al., 2014; SANTOS PS, et al., 2015).

Com relação a comunicação, foi apontada pelos profissionais a existência de dificuldades na comunicação entre os membros da equipe multidisciplinar no CC, entretanto, os profissionais reconheceram que o processo de comunicação se constitui uma importante ferramenta para desenvolvimento do trabalho e de adaptação frente as particularidades de cada equipe e/ou cirurgiões.

De acordo com a literatura são desafios encontrados para a comunicação efetiva e, consequentemente, para a segurança do paciente: falhas na comunicação na passagem de turnos e de informações entre os membros da equipe interdisciplinar, longas jornadas de trabalho, número inadequado de profissionais, divisões de tarefas e desigualdade nas relações de poder, aspectos também identificados no presente estudo (ALVES NB e ARAUJO GF, 2015; NOGUEIRA JWS e RODRIGUES MCS, 2015; FARIAS ES, et al., 2018).

A comunicação efetiva foi instituída como uma meta de segurança pelo Ministério da Saúde (MS) por meio da portaria $n^{\circ}$ 529, de 1ำ de abril de 2013, para obter uma comunicação de qualidade e, consequentemente, proporcionar a assistência ao paciente livre de danos. Esta meta deve ser trabalhada nos serviços de saúde, sendo necessário a observância de elementos fundamentais: olhar diretamente, escuta, compreender a mensagem, desenvolvimento da liderança, união de todos os membros da equipe e troca de informações para garantir uma assistência de qualidade (NOGUEIRA JWS e RODRIGUES MCS, 2015).

Diante disto, a comunicação além de necessária para uma assistência segura, passa a ter a função de promover o relacionamento entre as pessoas. A comunicação, seja verbal ou não-verbal, é um elo importante entre as equipes, assim, deve haver coparticipação entre emissor e receptor na busca de soluções proporcionando um canal de entendimento, viabilizando mudanças de comportamento e melhora nas relações interpessoais (MARTINS CCF, et al., 2014; STUMM EMF, et al., 2006).

Entre as limitações do estudo, destaca-se ter sido restrito a apenas uma unidade de centro cirúrgico do referido hospital universitário e a impossibilidade da participação de todos os profissionais das equipes cirúrgicas da unidade nos encontros, em virtude da rotina de trabalho e escalas de serviço dos mesmos. Contudo, a importância deste estudo está em revelar os principais problemas vinculados a comunicação e relacionamento interpessoal vivenciados pela equipe, assim como a promoção da mudança da realidade estudada.

\section{CONSIDERAÇÕES FINAIS}

Este estudo possibilitou a reflexão sobre as dificuldades vinculadas à comunicação e ao relacionamento interpessoal entre equipe multiprofissional no centro cirúrgico de um hospital universitário, bem como, permitiu discutir, teorizar e confrontar com a literatura pertinente os problemas identificados a partir da formulação de soluções e mudança da realidade. Os principais problemas apontados na comunicação e relacionamento interpessoal foram: dificuldades no relacionamento entre os membros da equipe; desvalorização do trabalho do enfermeiro; comunicação deficiente ou falha na comunicação; formação de "guetos" profissionais e sobrecarga de trabalho e funções. O Arco de Maguerez mostrou-se uma metodologia ativa eficiente ao aproximar teoria e prática com a problematização da realidade observada. Identificou-se o desenvolvimento do pensamento crítico-reflexivo por parte da equipe cirúrgica e maior conscientização acerca da importância da manutenção de uma boa comunicação e relacionamento interpessoal entre os profissionais que ali atuam, o que contribuiu para o fortalecimento das relações entre equipe multiprofissional. 


\section{REFERÊNCIAS}

1. ALVES NB, ARAUJO GF. Percepção do estresse entre os profissionais de enfermagem que atuam no centro cirúrgico de um hospital privado em vitória da Conquista-BA. Cad. Saúde Pública, 2015;5(1):77-88.

2. AMESTOY SC, et al. Gerenciamento de conflitos: desafios vivenciados pelos enfermeiros-líderes no ambiente hospitalar. Ver Gaúcha Enferm, 2014;35(2):79-85.

3. BERBEL NAN. A problematização e a aprendizagem baseada em problemas: diferentes termos ou diferentes caminhos? Interface: comunic, saude, educ, 1998;2(2):139-154.

4. CARVALHO R, BIANCHI ERF. Enfermagem em Centro Cirúrgico e Recuperação. $2^{\mathrm{a}}$ ed. Barueri: Manole, 2016; 428p.

5. CIAMPONE MHT, KURCGANT P. Gerenciamento de conflitos e negociação. In: KURCGANT P. Gerenciamento dos serviços de enfermagem. Rio de Janeiro: Guanabara Koogan, 2005; p. 54-65.

6. CORRADI EM et al. O gerenciamento de conflitos entre a equipe de enfermagem. Cogitare Enferm, 2008;13(2):184-93.

7. FARIAS ES, et al. Comunicação efetiva: elo na segurança do paciente no âmbito hospitalar. Cadernos de Graduação, 2018;4(3):139-157.

8. MARTINS CCF, et al. Relacionamento Interpessoal da Equipe de Enfermagem x Estresse: limitações para a prática. Cogitare Enferm, 2014;19(2):309-15.

9. MARTA CB, et al. Gestão de conflitos: competência gerencial do enfermeiro. R. pesq.: cuid. fundam. online 2010;2(Ed. Supl.):604-608.

10. NOGUEIRA JWS, RODRIGUES MCS. Comunicação efetiva no trabalho em equipe em saúde: um desafio para a segurança do paciente. Cogitare Enferm, 2015;20(3):636-640.

11. OLIVEIRA MAN, SANTA ROSA DO. Conflitos e dilemas éticos: vivências de enfermeiras no centro cirúrgico. Rev baiana enferm, 2016;30(1):344-355.

12. SANTOS KCB, et al. Metodologia da problematização com Arco de Maguerez no centro cirúrgico oftalmológico de um hospital universitário. ReonFacema, 2018;4(1):884-888.

13. SANTOS PS, et al. Relação entre médicos e enfermeiros do Hospital Regional de Cáceres Dr. Antônio Fontes: a perspectiva do enfermeiro. Rev Ciênc Estud Acad Med, 2015;4(1):10-28.

14. STUMM EMF, et al. Dificuldades enfrentadas por enfermeiros em um centro cirúrgico. Texto Texto contexto - enferm, 2006;15(3):464-471.

15. TRAJANO MFC, et al. Relações interpessoais no centro cirúrgico sob a ótica da enfermagem: estudo exploratório. Online Braz JNurs, 2017; 16(1):159-169. 